\title{
EL SAPO ARLEQUÍN DEL CHOCÓ (ATELOPUS SPURRELLI BOULENGER, 1914) EN EL PARQUE NACIONAL NATURAL UTRÍA, COLOMBIA: DENSIDAD POBLACIONAL Y RECOMENDACIONES PARA SU MONITOREO Y CONSERVACIÓN THE CONDOTO STUBFOOT TOAD (ATELOPUS SPURRELLI BOULENGER, 1914) AT UTRÍA NATIONAL PARK, COLOMBIA: POPULATION DENSITY MONITORING AND CONSERVATION Ángela Milena Melo-Beltrán ${ }^{1}$, Lincoln de Jesús Moya-Córdobaํ, Fabian Eliecer Vergara-Rosario ${ }^{1}$ of Diego A. Gómez-HoYos ${ }^{2,3 *}$ \\ ${ }^{1}$ Parques Nacionales Naturales de Colombia, PNN Utría, Territorial Pacífico, Calle 29 Norte No. 6N-43 Barrio Santa Mónica, Cali, Colombia. ${ }^{2}$ ProCAT Internacional/Sierra to Sea Institute Costa Rica, Las Alturas, Puntarenas, Costa Rica. \\ ${ }^{3}$ Grupo de Investigación y Asesoría en Estadistica/Grupo de Herpetología, Universidad del Quindío, Carrera 15 Calle 12 Norte, Armenia, Colombia. *Correspondencia: biodiego88@gmail.com
}

Abstract.- We assessed the population density of Atelopus spurrelli in Utría National Natural Park to establish a baseline that could be used in a future surveillance and monitoring program for the species, as well as to assess the effect of potential threats to this population. We surveyed seven transects of variable length in three streams with previous records of the species in the Natural Park and estimated population density using the distance sampling method and the Multinomial-Poisson Mixture model. We recorded 191 individuals of A. spurrelli with a density of 0.09 individuals $/ \mathrm{m}^{2}(95 \% \mathrm{CI}: 0.07-0.12)$ in Cocalito creek, 0.13 individuals $/ \mathrm{m}^{2}(0.1-0.18)$ in La Aguada creek and 0.14 individuals $/ \mathrm{m}^{2}$ (0.10-0.19) in Guachalito. Cocalito creek had the lowest estimated average density, which suggests biophysical or anthropic factors that vary between the creeks might drive this population parameter. We do not know exactly which factors affect the differences in population density between creeks in this study and other previous studies of the same population, but it could be attributed to the method of data analysis, the sampling representativity, or responses to biophysical and anthropic factors (e.g. human transit intensity) that vary between the creeks sampled. Thus, we suggest monitoring the populations multiple times a year for several years to determine precisely which factors drive the species' density estimations.

Keywords.- Atelopus, Bufonidae, density, imperfect detection, monitoring, threatened species.

Resumen.- Evaluamos la densidad poblacional de Atelopus spurrelli en el Parque Nacional Natural Utría para generar la línea base que permita establecer un programa de seguimiento y monitoreo de la especie, así como evaluar el efecto de potenciales amenazas sobre esta población. Seleccionamos tres quebradas con registros previos de la especie en el Parque Natural e inspeccionamos siete transectos de longitud variable. Estimamos la densidad poblacional con el método de muestreo por distancias usando el modelo Multinomial-Poisson Mixture. Registramos un total de 191 individuos de A. spurrelli con una densidad de 0.09 individuos $/ \mathrm{m}^{2}$ (IC $95 \%$ : 0.07-0.12) en la quebrada Cocalito, 0.13 (0.1-0.18) en la quebrada La Aguada y $0.14(0.10-0.19)$ en Guachalito. La quebrada Cocalito presentó la más baja densidad promedio estimada, lo que sugiere un factor biofísico o antrópico diferencial entre quebradas con un efecto sobre este parámetro poblacional. Desconocemos el factor relacionado con las diferencias en la densidad de la especie entre quebradas y en comparación a estudios previos de la misma población, pero podría atribuirse a artefactos de los métodos de análisis de los datos, la representatividad del muestreo o la respuesta a factores físicos y antrópicos (e.g. intensidad de tránsito humano) presentes de manera diferencial en las quebradas muestreadas. Por lo tanto, sugerimos realizar el seguimiento de la población en varios periodos del año y multianual para determinar los factores que operan sobre las estimaciones de densidad de la especie. 
Palabras clave.- Atelopus, Bufonidae, densidad, detección imperfecta, especie amenazada, monitoreo.

\section{INTRODUCCIÓN}

El género Atelopus está comprendido por 96 especies reconocidas (Frost, 2019), de las cuales el 83\% se encuentran en alguna categoría

de amenaza según la IUCN (2019). Muchas de estas especies no han sido observadas por décadas y podrían estar potencialmente extintas (e.g. Atelopus quimbaya, Gómez-Hoyos et al., 2018a). Por esta razón, los sapos arlequín se consideran como uno de los grupos de anfibios más amenazados a nivel mundial (Lötters, 2007). Su grado de amenaza se debe a la acción sinérgica de factores -particulares para el contexto de cada especie de Atelopus- como las enfermedades (e.g. quitridiomicosis), la pérdida y degradación del hábitat, el cambio climático, el comercio, las especies invasoras y la contaminación ambiental (La Marca et al., 2005).

Evaluaciones recientes del Grupo de Especialista de Anfibios, Comisión de Supervivencia de Especies de la IUCN (IUCN, 2019), muestran un panorama crítico para este grupo de anfibios. Sólo cuatro especies están categorizadas como en Preocupación Menor y otras dos como Casi Amenazadas, y en general se considera que sólo cinco especies tienen poblaciones estables (IUCN, 2019). Por otra parte, existen ocho especies categorizadas con Datos Insuficientes que evidencia las necesidades de conocimiento del estado de conservación de estos sapos arlequín en particular. En general, existen pocos estudios poblacionales a largo plazo que permitan determinar con certeza la supuesta estabilidad de algunas poblaciones o el estado poblacional de los sapos arlequín en general.

Algunas especies como Atelopus cruciger, A. ignescens, A. varius y A. zeteki cuentan con estudios poblacionales robustos (Ron et al., 2003; Lampo et al., 2012; 2017; McCaffery et al., 2015; GonzálezMaya et al., 2018, Gómez-Hoyos et al., 2020) que han permitido estimar parámetros demográficos y evaluar tendencias poblacionales, así como identificar amenazas y su efecto sobre las poblaciones. Sin embargo, la mayoría de sapos arlequín no cuentan con estudios poblacionales de línea base y para unas pocas especies como A. elegans y A. spurrelli solo se cuenta con evaluaciones poblacionales rápidas (Gómez-Hoyos et al., 2014; Gómez-Hoyos et al., 2017) y no es posible conocer su tendencia. Esta situación ha dificultado conocer con certeza las categorías de amenaza y la tendencia de la mayoría de poblaciones de los sapos arlequín. Las recientes evaluaciones están basadas en criterio experto que usualmente se apoyan en observaciones anecdóticas de las poblaciones de estas especies.
El sapo arlequín del Chocó (Atelopus spurrelli Boulenger, 1914) se consideró hasta el 2017 como Vulnerable y se pensaba que sus poblaciones estaban decreciendo (Bolívar \& Lötters, 2004). Sin embargo, en la más reciente evaluación la especie se categorizó como Casi Amenazada y con poblaciones estables (IUCN SSC Amphibian Specialist Group, 2017). Los criterios para el cambio de categoría fueron la extensión de su distribución y que la especie es localmente común en varios lugares, pero no se descarta que una aparente reducción en su área de ocurrencia permita su categorización como amenazada en el futuro cercano (IUCN SSC Amphibian Specialist Group, 2017). A pesar de esta información y criterios, a la fecha no se tiene conocimiento acerca de la dinámica y tendencia de sus poblaciones, así como tampoco de los efectos de factores biofísicos o antrópicos que puedan estar influyendo en estas.

Existe una población del sapo arlequín del Chocó (A. spurrelli) registrada en el Parque Nacional Natural Utría (PNN Utría; Gómez-Hoyos et al., 2014) la cual representa una oportunidad de investigación y conservación para la especie. Sin embargo, a la fecha sólo cuenta con un estudio poblacional rápido donde se estimó su densidad general (Gómez-Hoyos et al., 2014). Luego de la primera evaluación de línea base que se realizó en 2012 (Gómez-Hoyos et al., 2014), el área protegida decidió retomar el seguimiento de la densidad de la población en quebradas del parque, pues es necesario iniciar un programa de seguimiento y monitoreo que permita entender los factores que tienen efecto sobre la población, así como planear y ejecutar medidas de conservación.

En este estudio se presenta una experiencia reciente sobre la evaluación poblacional del sapo arlequín del Chocó (Atelopus spurrelli) en el PNN Utría en Colombia, implementada por el personal del área protegida en tres quebradas. También, se ofrecen algunas recomendaciones para fortalecer el seguimiento de esta población e identificar factores que tengan efecto sobre ésta, con el fin de planear y ejecutar acciones de conservación e iniciar un programa de monitoreo.

\section{MATERIALES Y MÉTODOS}

\section{Área de estudio}

El PNN Utría se encuentra ubicado en el departamento de 
Chocó en Colombia, en jurisdicción de los municipios de Bahía Solano, Nuquí, Alto Baudó y Bojayá (BirdLife International, 2019). El Parque se localiza entre $8.87^{\circ}-6.15^{\circ} \mathrm{N}$ y $77.22^{\circ}-$ $77.38^{\circ} \mathrm{W}$ y presenta un gradiente de elevación desde 0 hasta $1140 \mathrm{msnm}$, cubriendo un área total de 64328 ha de las cuales aproximadamente $88 \%$ son terrestres (Vásquez \& Serrano, 2009).

El estudio lo realizamos en la Ensenada de Utría (Fig. 1) zona que presenta una temperatura que oscila entre $27^{\circ} \mathrm{C}$ y $30^{\circ} \mathrm{C}$, con humedad relativa de $90 \%$ y una precipitación promedio multianual de $10000 \mathrm{~mm}$, que corresponde a formaciones vegetales de selva húmeda tropical (Vásquez \& Serrano, 2009). Los muestreos se realizaron en las Quebradas Cocalito $\left(6.02017^{\circ}\right.$ $\left.\mathrm{N}, 77.35531^{\circ} \mathrm{W}\right)$, La Aguada $\left(6.01142^{\circ} \mathrm{N}, 77.34081^{\circ} \mathrm{W}\right)$ y Guachalito $\left(6.00322^{\circ} \mathrm{N}, 77.33722^{\circ} \mathrm{W}\right)$.

\section{Diseño de muestreo}

Realizamos el muestreo por iniciativa de funcionarios del PNN Utría (autores AM, LM, FV; basado en las recomendaciones de Gómez-Hoyos et al., 2014) entre el 8 y 11 de marzo de 2019. Durante el día, entre las 8:00 y las 15:00 horas, inspeccionamos los cursos de tres quebradas a lo largo de 7 transectos de longitud variable (quebrada Cocalito: tres transectos de 131, 133 y $160 \mathrm{~m}$; quebrada La Aguada: tres transectos de 120, 121 y 118 m; quebrada Guachalito: un transecto de $155 \mathrm{~m}$ ), en busca de individuos de A. spurrelli con un esfuerzo total de 37.4 horas/persona (Cocalito: 11.25 horas/persona; La Aguada: 15.9 horas/persona; Guachalito: 10.25 horas/persona). Registramos a cada individuo observado la distancia perpendicular desde su ubicación hasta la orilla de la quebrada y se asignó a una categoría de edad. La categoría de edad la asignamos con el cálculo de intervalos discretos de tamaño a través de observación directa y comparación con objetos de referencia. Realizamos este procedimiento para evitar el contacto directo por manipulación o uso de herramientas de medición, de manera que se minimizara el potencial contagio de enfermedades ante la ausencia de un protocolo de bioseguridad durante el estudio. Las categorías de edad incluyeron inmaduros (<25 mm) y adultos ( $>25 \mathrm{~mm}$; Lötters, 1996), pero existe

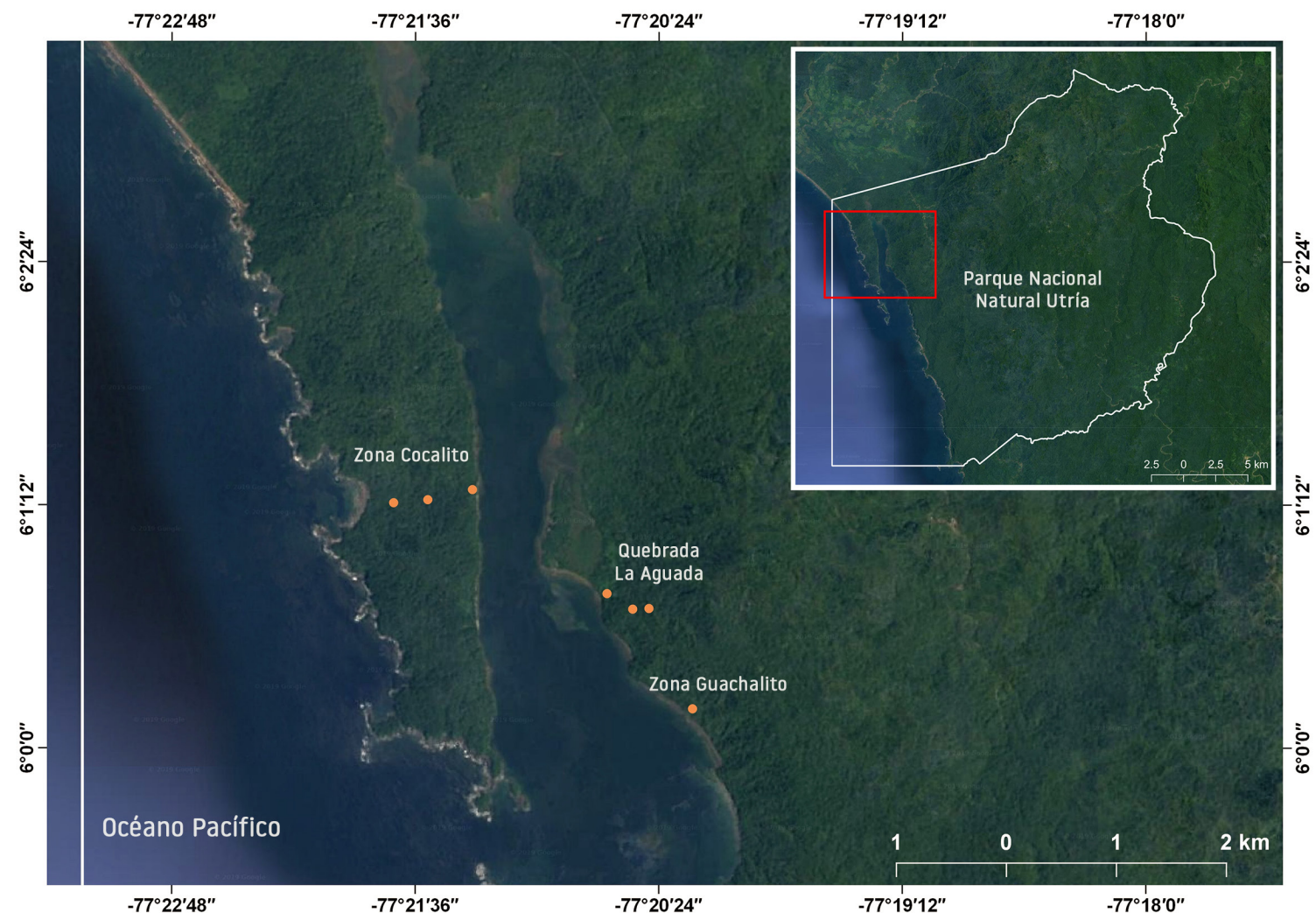

Figure 1. Location of the Utría National Natural Park and the areas evaluated to estimate the density of Atelopus spurrelli. Orange dots represent the location of the inspected transects.

Figura 1. Ubicación geográfica del Parque Nacional Natural Utría y las zonas evaluadas para estimar la densidad de Atelopus spurrelli. Puntos naranjas representan la ubicación de los transectos inspeccionados. 
incertidumbre en la asignación debido a que no se midieron directamente los individuos.

\section{Análisis de datos}

Para estimar las densidades de Atelopus spurrelli en cada quebrada utilizamos el método de muestreo por distancias ajustado al modelo Multinomial-Poisson mixture (M-Pm) (Royle et al., 2004). Ajustamos el modelo M-Pm a transectos y distancias perpendiculares en intervalos discretos usando la función distsamp del paquete Unmarked (Fiske \& Chandler, 2015) en el lenguaje R (R Core Team, 2018). Estos modelos incluyeron las funciones de detección Half normal, Uniform, Hazard rate y Negative exponential en combinación con un modelo nulo para detección y densidad, así como también modelos donde estos parámetros están afectados por las quebradas. Los resultados fueron representados en gráficas construidas con el paquete ggplot2 (Wickham, 2016). Los datos y el código de análisis están disponibles en el repositorio GitHub (https://github.com/ biodiego88/Publicacion_densidad_Aspurrelli_PNNUtria).

Para seleccionar los modelos con mejor ajuste a los datos usamos el Criterio de Información de Akaike corregido para muestras pequeñas (AICc en inglés), en donde los modelos con menor valor de AICc indican el modelo más plausible (Burnham y Anderson, 2002). Realizamos la selección de los modelos con el paquete AICcmodavg (Mazerolle, 2017). Usamos el modelo con el mejor ajuste para estimar la densidad y la probabilidad de detección del sapo arlequín del Chocó.

\section{RESULTADOS}

Registramos un total de 191 individuos de Atelopus spurrelli de los cuales 62 fueron encontrados en la quebrada Cocalito, 81 en la quebrada la Aguada y 48 en Guachalito. De los individuos observados 97 fueron asignados a la categoría inmaduros (posiblemente 62 individuos postmetamorfos) y 94 a adultos. Observamos 4 parejas en amplexo.

El modelo con mejor ajuste a los datos de distancias perpendiculares representa la función de detección Hazard rate con la detección y la densidad del sapo arlequín del Chocó explicada por el tipo de quebrada (Tabla 1). De acuerdo a este modelo, la densidad del sapo arlequín del Chocó fue menor en la quebrada Cocalito con estimaciones de 0.09 individuos $/ \mathrm{m}^{2}$ (error estándar: 0.01; Intervalo de Confianza 95\%: 0.07-0.12) en comparación a 0.13 individuos $/ \mathrm{m}^{2}$ (ee: 0.02; IC 95\%: 0.1-0.18) en la quebrada La Aguada y 0.14 individuos $/ \mathrm{m}^{2}$ (ee: 0.03 ; IC $95 \%$ : 0.10-0.19) en Guachalito (Fig. 2). La probabilidad de detección fue menor en la quebrada La Aguada con 0.49 (ee: 0.11; IC 95\%:
Table 1. Multinomial-Poisson mixture models created to identify the model with the best fit to estimate the density of the Chocó harlequin toad (Atelopus spurrelli) in the Utría National Natural Park. K: parameters; AICc: corrected Akaike Information Criteria; qda: creek.

Tabla 1. Modelos Multinomial-Poisson mixture generados para identificar el modelo con mejor ajuste para estimar la densidad del sapo arlequín del Chocó (Atelopus spurrelli) en el Parque Nacional Natural Utría. K: parámetros; AICc: Criterio de Información de Akaike corregido; qda: quebrada.

\begin{tabular}{|c|c|c|c|c|c|c|}
\hline Modelo & Función & K & AICC & $\begin{array}{l}\text { Delta } \\
\text { AICc }\end{array}$ & $\begin{array}{c}\text { Peso } \\
\text { AlCcWt }\end{array}$ & $\begin{array}{c}\text { Peso } \\
\text { acumulado }\end{array}$ \\
\hline y(qda) p(qda) & Hazard rate & 7 & 60.94 & 0.00 & 1 & 1 \\
\hline y(null) p(null) & Hazard rate & 3 & 192.17 & 131.23 & 0 & 1 \\
\hline y(null) p(null) & $\begin{array}{l}\text { Negative } \\
\text { exponential }\end{array}$ & 2 & 192.68 & 131.74 & 0 & 1 \\
\hline y(null) p(qda) & $\begin{array}{l}\text { Negative } \\
\text { exponential }\end{array}$ & 4 & 201.00 & 140.07 & 0 & 1 \\
\hline y(qda) p(null) & $\begin{array}{l}\text { Negative } \\
\text { exponential }\end{array}$ & 4 & 204.19 & 143.25 & 0 & 1 \\
\hline y(null) p(null) & Half normal & 2 & 225.63 & 164.69 & 0 & 1 \\
\hline y(null) p(qda) & Half normal & 4 & 231.96 & 171.02 & 0 & 1 \\
\hline y(null) p(qda) & Hazard rate & 5 & 232.11 & 171.17 & 0 & 1 \\
\hline y(qda) p(null) & Half normal & 4 & 237.15 & 176.21 & 0 & 1 \\
\hline y(qda) p(null) & Hazard rate & 5 & 238.68 & 177.75 & 0 & 1 \\
\hline y(null) p(qda) & Uniform & 1 & 558.64 & 497.70 & 0 & 1 \\
\hline y(null) p(null) & Uniform & 1 & 558.64 & 497.70 & 0 & 1 \\
\hline y(qda) p(qda) & Uniform & 3 & 560.36 & 499.42 & 0 & 1 \\
\hline y(qda) p(null) & Uniform & 3 & 560.36 & 499.42 & 0 & 1 \\
\hline y(qda) p(qda) & $\begin{array}{l}\text { Negative } \\
\text { exponential }\end{array}$ & 6 & Inf & Inf & 0 & 1 \\
\hline y(qda) plqda) & Half normal & 6 & $\operatorname{lnf}$ & Inf & 0 & 1 \\
\hline
\end{tabular}




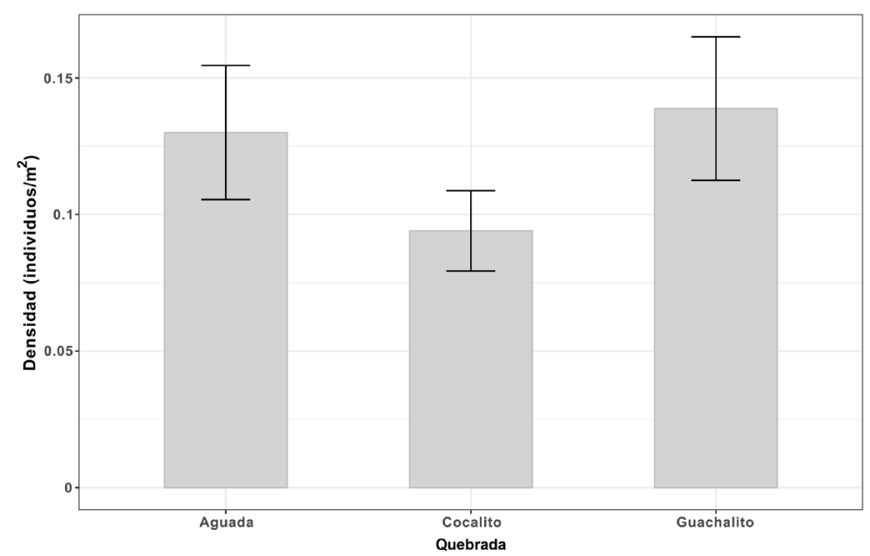

Figure 2. Population density estimation of the Chocó harlequin toad (Atelopus spurrelli) in three creeks of the Utría National Natural Park, Chocó, Colombia. The estimates were based on the model represented by the Hazard rate detection function and the detection probability and density depending on the creek evaluated. Error bars: 95\% Confidence intervals.

Figura 2. Estimación de la densidad poblacional del sapo arlequín del Chocó (Atelopus spurrelli) en tres quebradas del Parque Nacional Natural Utría, Chocó, Colombia. Las estimaciones estuvieron basadas en el modelo representado por la función de detección Hazard rate y la probabilidad de detección y densidad dependiendo de la quebrada evaluada. Barras de error: Intervalos de confianza $95 \%$

0.37-0.64) en comparación a 0.75 (ee: 0.1; IC 95\%: 0.6-0.93) en la quebrada Cocalito y 0.83 (ee: 0.11; IC 95\%: 0.66-1) en Guachalito (Fig. 3).

\section{DISCUSIÓN}

En el presente estudio evidenciamos una importante proporción de individuos inmaduros, así como algunas parejas amplectantes, tal como se registró en febrero de 2012 en el estudio realizado por Gómez-Hoyos et al. (2014), lo que sugiere actividad reproductiva durante o cercano al periodo de estudio. Sin embargo, es necesario darle continuidad al seguimiento del estado poblacional de la especie para determinar con certeza los periodos de actividad reproductiva de la especie en el PNN Utría.

En este estudio el modelo que mejor se ajustó a los datos de distancias perpendiculares estuvo representado por la función Hazard rate. Esta función es diferente a la encontrada en el estudio previo (Half-normal y Hermite polynomial; Gómez-Hoyos et al., 2014). Estas diferencias en la función de detección se deben posiblemente a los modelos que se usaron, en este estudio se utilizó el modelo M-Pm (Royle et al., 2004) y en el anterior se utilizó el muestreo por distancias convencional (Thomas et al., 2010).

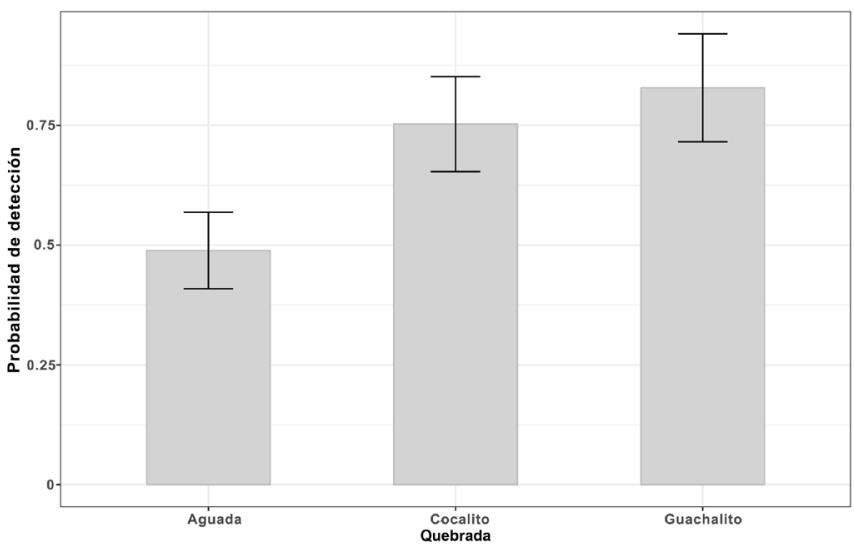

Figure 3. Detection probability estimation of the Chocó harlequin toad (Atelopus spurrelli) in three creeks of the Utría National Natural Park, Chocó, Colombia. The estimates were based on the model represented by the Hazard rate detection function. Error bars: 95\% Confidence intervals.

Figura 3. Estimación de la probabilidad de detección del sapo arlequín del Chocó (Atelopus spurrelli) en tres quebradas del Parque Nacional Natural Utría, Chocó, Colombia. Las estimaciones estuvieron basadas en el modelo representado por la función de detección Hazard rate. Barras de error: Intervalos de confianza $95 \%$

La densidad promedio del sapo arlequín del Chocó en el PNN Utría (entre 0.09 y 0.14 individuos $/ \mathrm{m}^{2}$ dependiendo de la quebrada) fue mayor a la calculada a partir de índices para Atelopus hoogmoedi en Suriname (Luger et al., 2008) y a la densidad de A. elegans en Colombia estimada con modelos de distancias perpendiculares (Gómez-Hoyos et al., 2017), y estuvo en el rango inferior de densidad calculado para $A$. varius en Costa Rica y fue menor a la densidad de A. ignescens en Ecuador (La Marca et al., 2005).

En comparación a la misma población estudiada en el 2012 (promedio de 0.03 individuos $/ \mathrm{m}^{2}$; Gómez-Hoyos et al., 2014) la densidad del sapo arlequín del Chocó fue mayor. Estas diferencias posiblemente se deben a la representatividad espacial de ambos estudios. En el estudio previo se muestrearon cinco quebradas y nueve transectos (ver Gómez-Hoyos et al., 2014) mientras que en el estudio actual se muestrearon tres quebradas y siete transectos. Estas diferencias en representatividad espacial de ambos estudios pueden influir tanto en las estimaciones de densidad obtenidas como en los modelos con mejor ajuste.

Por otra parte, no descartamos que las diferencias en las estimaciones de densidad de 2012 y las actuales se deban a que las aproximaciones de ambos modelos de distancias perpendiculares ( $M-P m$ y convencional) son diferentes: 1 ) en el 
muestreo por distancias convencional usualmente las distancias perpendiculares son exactas (supuesto del modelo; Thomas et al., 2010), mientras que en el multinomial $(\mathrm{M}-\mathrm{Pm})$ las distancias son intervalos discretos (Royle et al., 2004); 2) el modelo $M$ - $P m$ incluye un efecto de covarianza sobre la abundancia, mientras que el modelo convencional no lo implementa; 3) en el convencional para este caso en particular no se estratificó por tipo de quebrada (Gómez-Hoyos et al., 2014) mientras que en el multinomial la quebrada se usó como covariable para los parámetros de detección y densidad. A pesar de estas diferencias, un estudio que comparó ambas aproximaciones del muestreo por distancias para la Tangara Multicolor (Chlorochrysa nitidissima) encontró que los modelos con mejor ajuste representaban la misma función -Hazard rate-y las estimaciones de densidad eran similares pero con diferente precisión (Gómez-Hoyos et al., 2018b).

Por lo anterior, se sugiere realizar el seguimiento de la población en varios periodos del año y multianual, para determinar si las diferencias en la densidad de la especie son artefactos de los métodos de análisis y representatividad del muestreo o si realmente ocurren incrementos en la densidad de la población en la época del año muestreada. El seguimiento que se propone también ayudará a identificar los factores biofísicos y antrópicos que tengan efecto sobre la población. Para los factores antrópicos resaltamos la importancia de evaluar la intensidad de tránsito humano que ocurre sobre las quebradas muestreadas y que coincide con las estimaciones de densidad que obtuvimos (menor densidad en quebradas con mayor tránsito humano).

Con fines de un potencial seguimiento de esta población, presentamos algunos aspectos importantes para tener en cuenta:

Biología del sapo arlequín del Chocó: La densidad de la especie dependerá potencialmente de la época reproductiva, en la cual se espera que haya mayor agregación en las quebradas por ser su recurso para la reproducción (Lötters, 1996; Lötters, 2007; Gómez-Hoyos et al., 2014). Debido a que el parámetro que se está utilizando para el seguimiento poblacional es la densidad, es necesario disminuir la incertidumbre sobre la distribución espacial y agregación en sitios de reproducción para elegir los períodos en que se realizarán los seguimientos poblacionales multianuales. Para que sean comparables los periodos evaluados sugerimos que haya mayor representatividad espacial en el muestreo, incluyendo áreas por fuera de las quebradas. Esto es necesario para establecer de forma confiable la estabilidad, aumento o disminución de la densidad poblacional y su relación con variables ambientales, factores de amenaza o acciones de conservación.
Aproximaciones de análisis de datos: Para objetivos de seguimiento poblacional o programas de monitoreo, es necesario elegir modelos que arrojen estimaciones precisas y que la elección de las covariables tenga sentido biológico o causalidad. Por esta razón la aproximación de los análisis de datos es una parte fundamental en un programa de monitoreo. Como se discutió arriba, las aproximaciones del método de muestreo por distancias incluyen variaciones en su implementación de acuerdo al software o paquete (e.g. Distance: modelo convencional; unmarked: $M-P m)$. Por lo anterior, se recomienda el uso del modelo $\mathrm{M}-\mathrm{Pm}$ porque permite incluir un efecto de covarianza sobre la abundancia y se ha recomendado sobre el muestreo por distancias convencional para otras especies (Gómez-Hoyos et al., 2018b).

Por otra parte, el método por distancias en general permite obtener estimaciones de densidad con un esfuerzo menor relativo a otros métodos, pero no permite la estimación de parámetros demográficos. En la medida de lo posible, recomendamos un estudio demográfico utilizando métodos de captura-recaptura para conocer otros parámetros de la población como supervivencia, reclutamiento y crecimiento poblacional. Luego del estudio demográfico, será posible definir un método menos costoso para el seguimiento de la población y el programa potencial de monitoreo.

Estimaciones corregidas por detectabilidad: Para el seguimiento de esta población es necesario continuar con métodos que permitan la corrección de parámetros poblacionales por detectabilidad. El uso de índices de abundancia o densidad no son recomendados porque deben cumplir con los supuestos de detectabilidad constante y perfecta (Kéry et al., 2005). En este estudio y el de otras especies de Atelopus (e.g. Lampo et al., 2012 Gómez-Hoyos et al., 2014, 2017; McCaffery et al., 2015; GonzálezMaya et al., 2018) demuestran que la detectabilidad es menor a 1 y es heterogénea espacial y temporalmente, por lo tanto los supuestos de detectabilidad constante y perfecta no se pueden asegurar.

Entonces, es necesario usar modelos que corrijan los parámetros por detectabilidad, en el escenario de un seguimiento poblacional para esta especie y un programa de monitoreo en caso de que se propongan y ejecuten acciones de conservación para el Sapo Arlequín del Chocó.

Disponibilidad de datos y análisis: Existe un estudio previo realizado por Gómez-Hoyos et al. (2014) en el que estiman la densidad de Atelopus spurrelli en la misma localidad y con el mismo método de muestreo por distancias (con las diferencias 
y repercusiones de los diferentes modelos utilizados). Sin embargo, no fue posible acceder a los datos del estudio previo, ya que no fueron liberados con la publicación y no están disponibles por pérdida (Gómez-Hoyos obs. pers.).

Por esta razón, se recomienda que los datos e incluso el análisis sean liberados en repositorios (e.g. GitHub, GitLab) para permitir su reanálisis y reducir la incertidumbre en las comparaciones multianuales que se realicen en el seguimiento de la especie. Además, se evita la pérdida de información, se mitigan las barreras para el seguimiento de esta población y se respeta el principio de reproducibilidad de este tipo de estudios. Es posible que un potencial programa de conservación a largo plazo implique cambio de los investigadores involucrados y estos deben tener acceso y entender tanto los datos como las estimaciones previas.

Implicaciones para el área protegida: El área protegida proyecta realizar el seguimiento de la especie en las tres quebradas estudiadas, contemplando comparar temporada seca o de bajas lluvias (diciembre a abril) con la temporada de lluvia (mayo a noviembre) en diferentes años, lo que a su vez coincide con periodos de bajo y alto flujo turístico. Esto es importante para el área protegida en términos de la generación y ejecución de medidas de manejo pertinentes.

Es necesario verificar si las densidades de Atelopus spurrelli se relacionan con factores biofísicos o antrópicos (e.g. la intensidad de tránsito de personas por las quebradas durante visitas turísticas). Los resultados del presente estudio y su continuidad determinarán el protocolo y los indicadores a incluir en el programa de monitoreo del PNN Utría. El estudio realizado se desarrolló y se proyecta realizar con la participación permanente de funcionarios de planta del área protegida, lo que favorece su ejecución y adaptación a largo plazo. El seguimiento a esta población aporta información valiosa para suplir vacíos de conocimiento del Valor Objeto de Conservación (VOC) Selva Húmeda Tropical y sus componentes, y para la potencial selección de A. spurrelli como nuevo VOC de filtro fino. Para el área protegida es importante generar alianzas de investigación que permitan concertar un plan de investigación y conservación para la especie, lo cual requiere del compromiso institucional para la destinación de recursos humanos y financieros.

\section{CONCLUSIONES}

La densidad promedio estimada del sapo arlequín del Chocó fue más baja en la quebrada Cocalito en comparación a las demás quebradas, lo que puede indicar factores que están afectando la densidad de la especie. Estos factores pueden ser biofísicos, intrínsecos de la especie o de origen antrópico. Es necesario identificar los factores y la magnitud del efecto que tienen sobre los parámetros poblacionales.

Conocer aspectos de la biología e historia natural de Atelopus spurrelli es importante en escenarios de un programa potencial de seguimiento y monitoreo de la población del sapo arlequín del Chocó en el PNN Utría. Es importante conocer y definir la época reproductiva, así como las variaciones de la densidad poblacional en este periodo. Esto permitirá tener mayor certidumbre en la definición de los factores que afectan la densidad de la especie y la identificación de indicadores que midan la efectividad de potenciales acciones de conservación.

Agradecimientos.- Agradecemos al equipo del Parque Nacional Natural Utría por su apoyo, disposición y aportes para retomar el estudio de la población y por brindar el tiempo y medios logísticos necesarios para el levantamiento de la información de campo. Agradecemos a Lina Valencia de GWC y Maria José Camacho Durán de la Universidad Javeriana por sus aportes relevantes para mejorar este manuscrito. A Maria K. Stahl por la revisión y corrección del resumen en inglés.

\section{LITERATURA CITADA}

BirdLife International, 2019. Important Bird Areas factsheet: Parque Nacional Natural Ensenada de Utría. http://www.birdlife.org [consultado en abril 2019].

Bolívar, W. \& S. Lötters. 2004. Atelopus spurrelli. The IUCN Red List of Threatened Species 2004: e.T54556A11167055. Gland, Switzerland. http://dx.doi.org/10.2305/IUCN.UK.2004.RLTS. T54556A11167055.en [consultado abril 2019].

Burnham, K.P. \& D.R. Anderson. 2002. Model selection and multimodel inference: A practical information-theoretic approach. 2nd edition. Springer-Verlag, New York.

Fiske I. \& R. Chandler. 2011. Unmarked: An R Package for Fitting Hierarchical Models of Wildlife Occurrence and Abundance. Journal of statistical software 43:1-23.

Frost, D.R. 2019. Amphibian Species of the World: an Online Reference. Version 6.0. American Museum of Natural History, New York, USA. http://research.amnh.org/herpetology/ amphibia/index.html [consultado en abril de 2019]. 
Gómez-Hoyos, D.A., W. Bolívar-G, C.E. Burbano-Yandi \& J.L. García. 2014. Evaluación poblacional y estrategia de monitoreo para Atelopus spurrelli en el Parque Nacional Natural Utría, Colombia. Revista de Biodiversidad Neotropical 4:104-112.

Gómez-Hoyos, D.A., T. Suarez-Joaqui, W. Bolívar \& J.L. García. 2017. Population assessment strategy for Atelopus elegans (Bufonidae) in the Gorgona National Natural Park, Colombia. North-Western Journal of Zoology 13:154-158.

Gómez-Hoyos, D.A., W. Cardona, G.A. González-Durán, S.V. Flechas, G.H. Kattan \& J.A. Velasco. 2018a. The population decline of Atelopus quimbaya (Anura: Bufonidae) in the Andes of Colombia. Revista Latinoamericana de Herpetología 1:34-42.

Gómez-Hoyos, D.A., O.H. Marín-Gómez \& Y.L. Caicedo Ortiz. 2018b. Multinomial-Poisson mixture models reveal unexpected higher density estimates of an Andean threatened bird. BioRxiv pre-print; 11 abril.

Gómez-Hoyos, D.A., R. Seisdedos-de-Vergara, J. Schipper, R. Allard \& J.F. González-Maya. 2020. Potential effect of habitat disturbance on reproduction of the critically endangered harlequin frog Atelopus varius in Las Tablas, Costa Rica. Animal Biodiversity and Conservation 43.1:1-7.

González-Maya, J.F., D.A. Gómez-Hoyos, I. Cruz-Lizano \& J. Schipper. 2018. From hope to alert: demography of a remnant population of the Critically Endangered Atelopus varius from Costa Rica. Studies on Neotropical Fauna and Environment 53:194-200.

IUCN SSC Amphibian Specialist Group, 2017. Atelopus spurrelli. The IUCN Red List of Threatened Species 2017: e.T54556A49538284. Gland, Switzerland. http://dx.doi.org/10.2305/IUCN.UK.2017-3. RLTS.T54556A49538284.en. [consultado en abril de 2019].

IUCN, 2019. The IUCN Red List of Threatened Species. Version 20191. http://www.iucnredlist.org [consultado en Abril de 2019].

Kéry, M.J., A. Royle \& H. Schmid. 2005. Modeling avian abundance from replicated counts using binomial mixture models. Ecological applications 15:1450-1461.

La Marca, E., K.R. Lips, S. Lötters. R. Puschendorf, R. Ibáñez, J.V. Rueda-Almonacid, R. Schulte, C. Marty, F. Castro, J. ManzanillaPuppo, J.E. García-Pérez, F. Bolaños, G. Chaves, J.A. Pounds, E. Toral \& B.E. Young. 2005. Catastrophic population declines and extinctions in Neotropical harlequin frogs (Bufonidae: Atelopus). Biotropica 37:190-201.

Lampo, M., J. Celsa Señaris, A. Rodríguez-Contreras, F. RojasRunjaic \& C.Z. García. 2012. High turnover rates in remnant populations of the harlequin frog Atelopus cruciger (Bufonidae): Low risk of extinction? Biotropica 44:420-426.

Lampo, M., J. Celsa Señaris \& C.Z. García. 2017. Population dynamics of the critically endangered toad Atelopus cruciger and the fungal disease chytridiomycosis. PloS one 12:e0179007.

Lötters, S. 1996. The neotropical toad genus Atelopus. Checklist - Biology - Distribution. Köln: M Vences \& Glaw Verlags.

Lötters, S. 2007. The fate of the harlequin toads-help through a synchronous multi-disciplinary approach and the IUCN. Amphibian conservation action plan? Zoosystematics and Evolution 83:69-73.

Mazerolle, M.J. 2017. AICcmodavg: Model selection and multimodel inference based on (Q)AIC(c). R package version 2.1-1. https:// cran. $r$-project.org $/$ package $=$ AICcmodavg

McCaffery, R., C.L. Richards-Zawacki \& K.R. Lips. 2015. The demography of Atelopus decline: Harlequin frog survival and abundance in central Panama prior to and during a disease outbreak. Global Ecology and Conservation 4:232-242.

R Core Team, 2018. R: A language and environment for statistical computing. R Foundation for Statistical Computing, Vienna, Austria. https://www.R-project.org/

Ron, S.R., W.E. Duellman, L.A. Coloma \& M.R. Bustamante. 2003. Population decline of jambato toad Atelopus ignescens (Anura: Bufonidae) in the Andes of Ecuador. Journal of Herpetology 37:116-126.

Royle, J.A., D.K. Dawson \& S. Bates. 2004. Modeling abundance effects in distance sampling. Ecology 85:1591-1597.

Thomas, L., S.T. Buckland, E.A. Rexstad, J.L. Laake, S. Strindberg, S.L. Hedley, J.R.B Bishop, T.A. Marques \& K.P. Burnham. 2010. Distance software: design and analysis of distance sampling surveys for estimating population size. Journal of Applied Ecology 47:5-14. 
Vásquez, V.H. \& M.A. Serrano. 2009. Las áreas naturales protegidas de Colombia. 1 de. Bogotá: Conservación Internacional, Colombia y Fundación Biocolombia.
Wickham, H. 2016. ggplot2: Elegant Graphics for Data Analysis. Springer-Verlag New York.

to 